\title{
The effect of cerebellar cortical degeneration on adaptive plasticity and movement control
}

\author{
Susen Werner · Otmar Bock · Dagmar Timmann
}

Received: 27 February 2008/ Accepted: 3 October 2008/Published online: 24 October 2008

(C) The Author(s) 2008. This article is published with open access at Springerlink.com

\begin{abstract}
Clinical and neuroimaging studies provide converging evidence that the cerebellum plays an important role for sensorimotor adaptation by participating in the adaptive process per se, and/or by evaluating motor performance errors as a prerequisite for adaptation. Recent experimental evidence suggests that error signals pertinent to adaptation are related to sensory prediction rather than to online corrections (Tseng et al. in J Neurophysiol 98(1):54-62, 2007). To further elucidate the role of the cerebellum, the present study uses a multiple regression approach to separate out three independent determinants of adaptive success. Seventeen patients with cerebellar atrophy but without extra-cerebellar lesions, and 17 healthy, sex- and age-matched controls participated. Both subject groups performed center-out pointing movements before, during, and after exposure to $60^{\circ}$ rotated visual feedback. From the registered data, we quantified four indicators of adaptive success (adaptive improvement, retention without feedback, intermanual transfer, and de-adaptation under normal feedback), as well as five measures of motor performance (reaction time, peak velocity, movement time, response variability, and ability for online error corrections). The variance of each adaptation indicator was then partitioned into three components, one related to subject group but not to motor performance, a second related to
\end{abstract}

S. Werner $(\bowtie) \cdot$ O. Bock

Institute of Physiology and Anatomy, German Sport University, 50927 Cologne, Germany

e-mail:Werner@dshs-koeln.de

O. Bock

e-mail: bock@dshs-koeln.de

D. Timmann

Department of Neurology, University of Duisburg-Essen,

Essen, Germany group and motor performance, and a third related to motor performance but not to group. In accordance with previous work, adaptation and motor performance were degraded in patients. The deficit was similar in magnitude for all four adaptation indicators, which suggests that adaptive recalibration rather than strategic control were affected in our patients. No adaptation indicator shared statistically significant variance with group alone; we therefore found no evidence for cerebellar circuitry dedicated to adaptation but not motor performance. Three indicators shared significant variance jointly with group and motor performance; this suggests that the cerebellar contribution to motor performance is related to adaptive success. All four indicators shared significant variance with motor performance alone; this indicates that extracerebellar contributions to motor performance are also related to adaptive success. In conclusion, our data support the view that neural structures inside and outside the cerebellum are processing motor performance-related signals as a prerequisite for adaptation, but provide no evidence for a cerebellar structure related exclusively to adaptation.

Keywords Sensorimotor coordination - Cerebellum . Ataxia $\cdot$ Motor learning $\cdot$ Pointing

\section{Introduction}

Since the pioneering theoretical work of Albus (1971) and Marr (1969), the cerebellum has been considered a crucial brain structure for motor learning. Indeed, experimental evidence supports its contribution to the conditioning (Gerwig et al. 2003; Timmann et al. 2000; Woodruff-Pak 1997), habituation (Maschke et al. 2000), and scaling (Bloedel and Bracha 1997) of various reflexes. The 
cerebellum has also been implicated in a more complex form of motor learning, namely, sensorimotor adaptation to visual and mechanical distortions. This view is supported by clinical studies, which found that adaptation is often reduced or abolished in patients with cerebellar disease (Deuschl et al. 1996; Diedrichsen et al. 2005; Gauthier et al. 1979; Martin et al. 1996; Maschke et al. 2004; Tseng et al. 2007; Weiner et al. 1983). Further support comes from functional neuroimaging studies, which observed an increase of cerebellar activity during an adaptation task (e.g., Flament et al. 1996; Graydon et al. 2005; Imamizu et al. 2000; Krakauer et al. 2004; Krebs et al. 1998; Lang et al. 1988).

A long-standing debate in literature focuses on the specific role played by the cerebellum during adaptation. According to one position, this brain structure is involved in the adaptive process per se, by storing an internal model of body and surrounds, which can be adaptively modified to compensate for imposed external distortions. According to the alternative position, the cerebellum monitors and controls the execution of movements, and thus provides performance-related signals as a crucial prerequisite for adaptation. In support for the first view, cerebellar patients show adaptation deficits even when executing ballistic responses, which are too fast for online error monitoring (Deuschl et al. 1996; Martin et al. 1996; Maschke et al. 2004; Tseng et al. 2007). In support for the second view, cerebellar activation in healthy subjects is more closely associated with performance errors than with adaptive progress (Flament et al. 1996). Another study favors a compromise between both above positions: adaptation was associated with widely distributed cerebellar activation which gradually decreased with practice, but also with focussed activation near the posterior superior fissure which did not decrease with practice and which persisted even after equating for performance errors (Imamizu et al. 2000). The authors concluded that the distributed activation might reflect performance-related processes, while the focussed activation might reflect the internal model.

A recent study (Tseng et al. 2007) compared adaptation under two conditions: when subjects executed ballistic movements which did not allow online error corrections, and when they performed slower movements which did allow such corrections. They found no difference between conditions in healthy subjects, which indicates that adaptation is not driven by online response corrections, but rather by the mismatch between intended and perceived response, called "sensory prediction error". They also found no difference between conditions in cerebellar patients, which suggests that patients' adaptation deficits cannot be explained by impaired online corrections. This outcome doesn't distinguish between the above two positions, but it stipulates that performance-related signals provided by the cerebellum for adaptation would include sensory predictions rather than online corrections.

To further elucidate the role of the cerebellum, the present study uses a multiple regression approach to separate out three components of adaptive success: one related to cerebellar integrity but not to motor performance, the second related jointly to cerebellar integrity and motor control performance, and the third to motor performance but not cerebellar integrity. A significant contribution of the first component would support the existence of cerebellar circuitry dedicated to adaptive processing but not to motor performance. A significant contribution of the second component would reflect cerebellar mechanisms involved in the monitoring and control of movementsincluding sensory prediction, while a significant contribution of the third component would support the role of mechanisms for motor control which are spared in cerebellar degeneration.

\section{Material and methods}

\section{Subjects}

Seventeen patients and 17 control subjects participated after providing written informed consent. Both groups were matched in age (patients $55.29 \pm 10.34$ years, control $54.59 \pm 8.57$ years) and gender ( 8 females and 9 males each). All participants were right-handed, and took no medicine affecting the central nervous system. All control subjects were in good health, and had no history of neurological disease. Patients presented with forms of cerebellar cortical degeneration, that is, sporadic adult onset ataxia (SAOA), spinocerebellar ataxia type 6 (SCA6) and genetically undetermined autosomal dominant cerebellar ataxia type III (ADCA III). Magnetic resonance imaging (MRI) revealed cerebellar atrophy with no extracerebellar lesions in all patients. Clinical examination showed a pure cerebellar syndrome in the majority of cases, with mild accompanying pallhypesthesia and/or hyperreflexia of the lower limbs in seven patients. Each patient's diagnosis, severity of ataxia, and extent of cerebellar atrophy are provided in Table 1 . On the average, cerebellar volume was $7.1 \pm 1.5 \%$ of total intracranial volume in female, and $7.1 \pm 0.7 \%$ in male patients; the corresponding values for healthy individuals are $8.2 \pm 0.7 \%$ in females and $8.0 \pm 0.7 \%$ in males (Dimitrova et al. 2006). The cerebellar volume of individual patients correlated inversely with their severity of ataxia $(r=-0.67, p<0.05)$. The experimental protocol was preapproved by the authors' local Ethics Committee. 
Table 1 Patients' characteristics

\begin{tabular}{lllllll}
\hline Patient & Age & Sex & Diagnosis & Volume & \multicolumn{2}{l}{ Ataxia } \\
\cline { 5 - 7 } & & & & & Total & Upper-limb \\
\hline HS & 66 & M & SAOA & $6.5^{*}$ & $20 / 56$ & $7 / 24$ \\
FS & 59 & F & SAOA & $7.2^{*}$ & $15 / 56$ & $5 / 24$ \\
MI & 66 & F & SAOA & 8.0 & $18 / 56$ & $7 / 24$ \\
EE & 45 & F & SAOA & $6.7^{*}$ & $19 / 56$ & $8 / 24$ \\
US & 39 & F & SAOA & $5.1^{*}$ & $23 / 56$ & $10 / 24$ \\
KT & 42 & M & ADCAIII & $6.4^{*}$ & $16 / 56$ & $5 / 24$ \\
DB & 48 & M & SAOA & $6.6^{*}$ & $19 / 56$ & $5 / 24$ \\
HG & 65 & M & SCA 6 & $7.2^{*}$ & $24 / 56$ & $10 / 24$ \\
HM & 67 & M & SAOA & 7.7 & $5 / 56$ & $2 / 24$ (left only) \\
DS & 49 & M & SCA 6 & 7.9 & $5 / 56$ & $2 / 24$ \\
PK & 65 & M & ADCAIII & 8.0 & $14 / 56$ & $6 / 24$ \\
PF & 47 & M & SAOA & $6.3^{*}$ & $14 / 56$ & $5 / 24$ \\
CW & 42 & F & ADCAIII & $4.7^{*}$ & $7 / 56$ & $5 / 24$ \\
RB & 53 & F & SAOA & 8.2 & $12 / 56$ & $4 / 24$ \\
WA & 56 & F & SCA6 & 7.6 & $18 / 56$ & $8 / 24$ \\
FR & 61 & F & ADCAIII & 9.0 & $0 / 56$ & $0 / 24$ \\
KF & 70 & M & SCA 6 & $6.8^{*}$ & $13 / 56$ & $3 / 24$ \\
\hline Age & & & & & &
\end{tabular}

Age, sex ( $F$ female, $M$ male), diagnose (ADAC III autosomal dominant cerebellar ataxia type III, SAOA sporadic adult onset ataxia, $S C A 6$ spinocerebellar ataxia type 6), cerebellar volume as percent of intracranial volume (asterisks mark values less than mean minus standard deviation of healthy individuals), and total as well as upperlimp ataxia scores from SARA (Schmitz-Hubsch et al. 2006). Note that subscores of upper-limb ataxia of the right and left arm were summed up, and no means were taken as in the original publication

\section{Procedure}

Seated subjects were instructed to point quickly and accurately at visual targets. As shown schematically in Fig. 1a, they watched a computer screen (S) through a mirror (M), such that the virtual image of the screen coincided with the horizontal surface of a digitizing tablet (T). A starting dot appeared for $0.5-3.0 \mathrm{~s}$ in the center of the virtual display, and was then replaced by one of eight possible target dots, located $45^{\circ}$ apart along an imaginary circle of $10 \mathrm{~cm}$ radius about the center; $2 \mathrm{~s}$ later the target was replaced by the starting dot, irrespective of how accurately the subjects had pointed. The starting dot remained on for $0.5-3.0 \mathrm{~s}$ then the next target appeared, etc. Subjects held a digitizing pen in their hand, and pointed at each target and back by moving the pen across the digitizing tablet. They were unable to see their arm, due to the mirror and surrounding shrouds; however, pen position was registered with a resolution of $0.3 \mathrm{~mm}$ and $60 \mathrm{~Hz}$, and was displayed on the screen as a cursor to provide visual feedback about instantaneous hand position. Thus, the subjects' task was essentially to move the cursor towards each target and back.

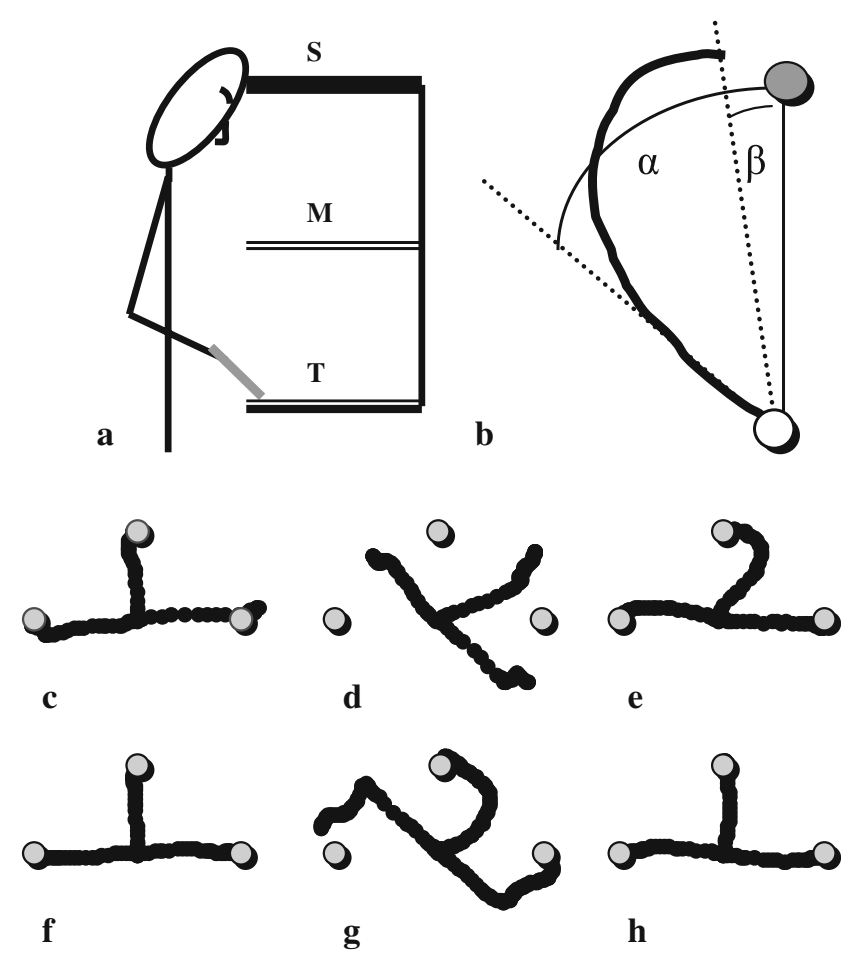

Fig. 1 a Scheme of experimental apparatus with display screen (S), mirror (M), and digitizing tablet (T). b Angles used for calculating the online correction ability (CA): White and gray dots represent starting point and target, the black line shows a sample movement path, $\alpha$ is the initial pointing error (150 ms after movement onset), $\beta$ the final error, and $(\alpha-\beta)$ is the correction angle. CA was calculated as the coefficient of determination between the correction angle and $\alpha$. c-e Sample cursor paths towards three of the eight possible targets in a patient with diffuse cerebellar atrophy, registered during the baseline phase (c), the beginning of the adaptation phase (d), and the end of the adaptation phase (e). $\mathbf{f}-\mathbf{h}$ Corresponding sample movement paths from a healthy control subject

The experiment was subdivided into episodes of $30 \mathrm{~s}$ duration, or about 6-12 target presentations, separated by rest breaks of about $4 \mathrm{~s}$. Depending on the particular episode, subjects used either their right or their left hand for pointing, and visual feedback about hand position was either veridical or rotated $60^{\circ}$ clockwise about the starting dot. The experiment began with one familiarization episode, in which subjects pointed with their right hand under veridical visual feedback. Next came the baseline phase, with one episode using the left, and then three episodes using the right hand, again under veridical feedback. The subsequent adaptation phase consisted of 20 episodes with the right hand, in which visual feedback was rotated by $-60^{\circ}$ to induce adaptation. Subjects returned 1 day or 1 week later ${ }^{1}$ for the retention phase of five episodes, using

\footnotetext{
1 The patients also took part in another study where different pause lengths were part of the test design. We did not anticipate an effect of pause length in our study, since retention of the adapted state remains nearly complete even after a 1-month pause (Bock et al. 2001).
} 
again the right hand under rotated feedback. Next came the intermanual transfer phase of two episodes, using the left hand under rotated feedback, followed by a single refresh episode, using the right hand under rotated feedback. Finally came the de-adaptation phase of five episodes, using the right hand under veridical feedback. The total testing time including instructions was about $20 \mathrm{~min}$ on the first, and about $10 \mathrm{~min}$ on the second day.

Data analysis

To quantify the time-course of adaptive improvement, we determined the initial error of each response as the angular difference between cursor and target direction $150 \mathrm{~ms}$ after response onset, i.e., before feedback-based corrections could become effective. Response onset was defined as the first sample after movement speed exceeded $32 \mathrm{~mm} / \mathrm{s}$; response end was determined accordingly. The mean initial errors of each subject and episode were submitted to an analysis of variance (ANOVAs) with the between-factor group (patient, control) and the within-factor episode; Huynh-Feldt-adjustments to the degrees of freedom were applied when necessary to compensate for heterogeneity of variances.

For further data reduction, we calculated each subject's adaptive success as adaptive improvement (AI), adaptive retention (AR), adaptive transfer (AT), and de-adaptation (DA)

$\mathrm{AI}\left({ }^{\circ}\right)=60-B+A$,

$\mathrm{AR}\left(^{\circ}\right)=60-B+\mathrm{R}$,

$\mathrm{AT}\left(^{\circ}\right)=60-B+\mathrm{T}$

$\mathrm{DA}\left({ }^{\circ}\right)=\mathrm{D}-B$,

where $B$ and $A$ are the mean initial error of the last three baseline and the last three adaptation episodes, respectively, and R, T, and D are the initial errors of the first retention, transfer, and deadaptation episode, respectively.

We further determined several measures of each subject's motor performance. To quantify the speed of responding, we calculated the means of reaction time (RT), peak velocity (PV), and movement time (MT) during the adaptation phase. To quantify the consistency of the initial, ballistic portion of responses, we calculated response variability (RV) as the standard deviation of initial errors about their respective mean. This measure was calculated only from the last three baseline episodes, to ensure that it is not contaminated by variability related to adaptive change.

To quantify the ability for online error corrections, we calculated the final error as the angular difference between cursor and target direction at the end of each movement, and defined the difference between initial and final errors as correction angle $(\alpha-\beta$ in Fig. 1b). Subjects with good correction ability should produce large correction angles when initial errors are high, and small correction angles when initial errors are low; in contrast, subjects with poor correction ability should produce correction angles, which are not closely related to initial errors. We therefore quantified each subject's correction ability (CA) as the coefficient of determination between correction angles and initial errors. To obtain robust values, we calculated CA from data of the adaptation phase, where initial errors were large; however, we excluded the very first adaptation episode, since some movements in that episode looked quite erratic. We considered it justifiable to determine CA, even though online corrections do not appear to affect adaptive success (Tseng et al. 2007), since CA could arguably reflect the processing of prediction errors as well (see "Introduction" and "Discussion").

The relationship between adaptive success, subject group, and motor performance was scrutinized with a multiple linear regression approach. To this end the total variance of each adaptation indicator (AI, AR, AT, DA) was partitioned into several components as shown in Fig. 2. The variance shared between the adaptation indicator $(A)$ and the group $(G)$ equals the coefficient of determination between $A$ and $G, R^{2}(G)$, represented in Fig. 2 by the common area $\operatorname{Var}_{G}+\operatorname{Var}_{J}$. Furthermore, the variance shared between $\mathrm{A}$ and the performance measures CA, RV, RT, PV, MT equals the multiple coefficient of determination $R^{2}(P)$, reflected in Fig. 2 by the common area $\operatorname{Var}_{J}+\operatorname{Var}_{P}$. Likewise, the variance shared between $\mathrm{A}, \mathrm{G}$, and $\mathrm{P}$ corresponds to the multiple coefficient of determination $R^{2}(P G)$, represented in Fig. 2 as $\operatorname{Var}_{G}+$ $\operatorname{Var}_{J}+\operatorname{Var}_{P}$. A detailed explanation of the partitioning of variances can be found in Bock and Girgenrath (2006).

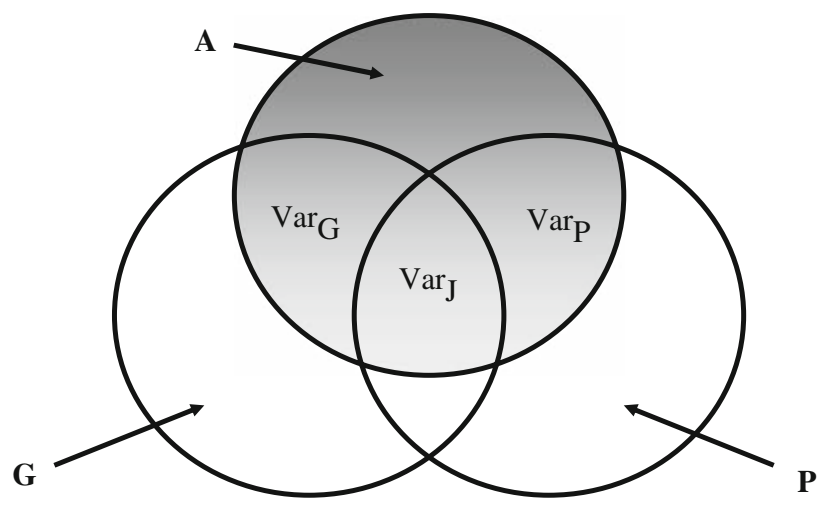

Fig. 2 The concept of common and unique variances. Circle $A$ represents the variance of an adaptation indicator AI, AR, AT, or DA, respectively. Circle $G$ pictures the variance of group and circle $P$, the shared variance of the performance measures $(\mathrm{CA}, \mathrm{RV}, \mathrm{RT}, \mathrm{PV}$, and MT). The overlapping areas indicate the variance of $A$ shared with $G$ only $\left(\operatorname{Var}_{G}\right)$, with $P$ only $\left(\operatorname{Var}_{P}\right)$, and with $G$ and $P$ jointly $\left(\operatorname{Var}_{J}\right)$ 
Consequently, the variance of $A$ can be partitioned into a component $\operatorname{Var}_{G}$ shared with group but not with motor performance, a component $\operatorname{Var}_{J}$ shared jointly with group and motor performance, and a component $\operatorname{Var}_{P}$ shared with motor performance alone:

$$
\begin{aligned}
& \operatorname{Var}_{G}=R_{P G}^{2}-R_{P}^{2}, \quad \operatorname{Var}_{J}=R_{G}^{2}+R_{P}^{2}-R_{P G}^{2}, \\
& \quad \operatorname{Var}_{P}=R_{P G}^{2}-R_{G}^{2},
\end{aligned}
$$

If $R_{P G}^{2}-R_{P}^{2}$ is significant as analyzed by the significance test of the Pearson product-moment correlation, then $\operatorname{Var}_{G}$ makes a decisive contribution to $A$.

\section{Results}

Figure 1 shows original registrations of cursor paths produced by a patient (c-e), and by a control subject (f-h). Paths registered during the baseline phase (c, f) are straight and well aimed. At the onset of adaptation (d, g), paths are misdirected by about $60^{\circ}$, as expected due to the imposed visual rotation; the paths curve back towards the targets later on in the control subject but not in the patient, suggesting that online error corrections are more pronounced in the control subject. Near the end of adaptation $(e, h)$, the paths become again straighter and more accurate, particularly in the control subject.

The above observations are confirmed and expanded by Fig. 3. The initial error across subjects from the control and the patient group was near zero during the baseline phase, became abruptly negative at the onset of adaptation, and then gradually returned towards zero, more so in controls than in patients. The group difference attained at the end of the adaptation phase persisted throughout the subsequent retention, transfer, and refresh phases, and then gradually decreased during the deadaptation phase. These observations were confirmed statistically. An ANOVA of the adaptation phase yielded significant effects of Group $(F(1,31)=18.78$; $p<0.001)$, Episode $(F(17,526)=45.19 ; p<0.001)$ and

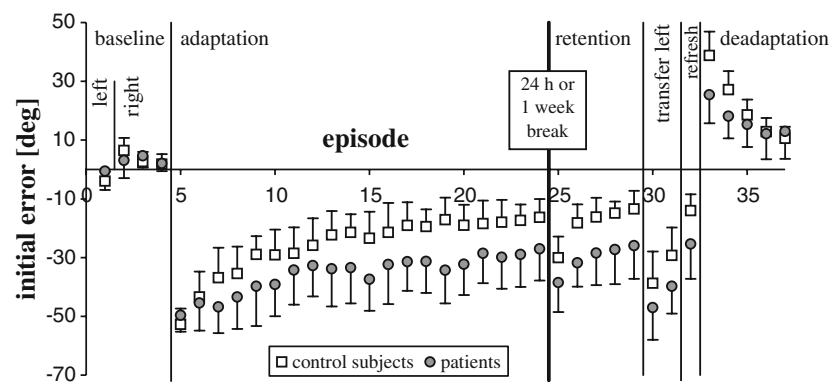

Fig. 3 Initial pointing error for all experimental episodes in patients (gray) and controls (white); left indicates episodes in which subjects used their left arm for pointing. Symbols represent across-subject means, and bars the pertinent standard deviations
Group*Episode $\quad(F(17,526)=3.71 ; \quad p<0.001) . \quad$ An ANOVA of episodes 22 to 33 (i.e., late adaptation to early deadaptation) yielded significant effects for Group $(F(1,32)=22.78 ; p<0.001)$ and Episode $(F(11,352)=$ 314.13; $p<0.001$ ) but not for their interaction. Finally, an ANOVA of the deadaptation phase yielded a significant effect of Group $(F(1,32)=5.22 ; p<0.05)$, Episode $(F(3,86)=111.90 ; \quad p<0.001)$ and their interaction $(F(3,86)=15.95 ; p<0.001)$.

Even though Bock et al. (2001) found the adapted state to remain nearly complete after a one-month pause, one might argue that this is not the case for patients. However, additional ANOVAs with the between-factors group (patient, control) and pause length (day, week) yielded no significant effects for pause length (Retention $F(1,30)=$ $0.00 ; \quad p>0.05 ; \quad$ Transfer $F(1,30)=0.07 ; \quad p>0.05$; Deadaptation: $F(1,30)=0.49 ; p>0.05)$, thus discarding the above argument.

The top part of Table 2 summarizes our findings regarding the four adaptation indicators. All indicators were significantly lower in patients than in control subjects, thus confirming the existence of adaptation deficits in cerebellar disease (see "Introduction"). Within the patient group, three indicators correlated significantly with the ataxia score, and one with cerebellar volume. The bottom part of Table 2 summarizes our findings regarding the five motor performance measures. Patients show significantly poorer performance than controls on three of those measures, and the same three measures were also significantly correlated with patients' ataxia scores. The correlation with cerebellar volume was not significant for any measure.

Table 3 summarizes the outcome of our multiple regression analyses. The variance shared with diagnosis but not motor performance $\left(\operatorname{Var}_{G}\right)$ was not significant for any adaptation indicator, the variance shared with diagnosis and motor performance $\left(\operatorname{Var}_{J}\right)$ was significant for three, and the variance shared with motor performance alone $\left(\operatorname{Var}_{P}\right)$ was significant for all four indicators. We noticed that some of our subjects' responses were slow, and terminated only after target disappearance, which could potentially bias our CA and MT scores. We, therefore, decided to replicate the regression analyses using only responses which terminated in time. One control subject and four patients had to be excluded from this replication because of too few acceptable movements. After sorting out all movements with $(\mathrm{MT}+\mathrm{RT})>2 \mathrm{~s}$ there was no more difference between groups for MT $(t(27)=-0.46$, $p>0.5$ ). However, the remaining data yielded exactly the same pattern of significant and non-significant variance components as in Table 3.

One might argue that our performance measures are not independent from adaptation since they were taken during the adaptation phase. We therefore recalculated CA, RT, 
Table 2 Indices of adaptation (top part) and measures of movement performance (bottom part) in healthy controls and in cerebellar patients

\begin{tabular}{lccccc}
\hline & Mean \pm SD & & $t(32)$ & \multicolumn{2}{c}{ Correlations with } \\
\cline { 2 - 4 } & Controls & Patients & & Patients' ataxia & Patients' volume \\
\hline AI & $39.2 \pm 5.4$ & $28.1 \pm 9.2$ & $-4.3^{* * *}$ & $-0.674^{*}$ & $0.348^{*}$ \\
AR & $26.3 \pm 7.7$ & $18.2 \pm 10.5$ & $-2.5^{*}$ & $-0.443^{*}$ & 0.166 \\
AT & $17.7 \pm 11.4$ & $9.7 \pm 10.8$ & $-2.1^{*}$ & -0.303 & 0.123 \\
DA & $35.1 \pm 8.2$ & $22.2 \pm 10.0$ & $-4.1^{* * *}$ & $-0.534^{*}$ & 0.306 \\
CA & $0.9 \pm 0.1$ & $0.7 \pm 0.3$ & $-3.2^{* *}$ & $-0.515^{*}$ & 0.077 \\
RV & $11.1 \pm 8.0$ & $14.6 \pm 11.6$ & 1.0 & 0.112 & 0.080 \\
RT & $0.4 \pm 0.1$ & $0.5 \pm 0.1$ & $3.3^{* *}$ & $0.556^{*}$ & -0.069 \\
PV & $246.8 \pm 64.6$ & $202.6 \pm 77.6$ & -1.8 & -0.237 & 0.163 \\
MT & $1.1 \pm 0.2$ & $1.4 \pm 0.4$ & $2.2^{*}$ & $0.355^{*}$ & -0.211 \\
\hline
\end{tabular}

The third data column presents the outcome of group comparisons with $t$ tests, and the last two columns are the correlations of patients' findings with ataxia scores and cerebellar volume. Symbols $* * *, * *$, and $*$ indicate $p<0.001, p<0.01$, and $p<0.05$, respectively, and the absence of a symbol indicates $p>0.05$

PV, and MT using the baseline phase, and repeated the multiple regression analysis: the significance pattern did not change, which suggests that our performance measures were not corrupted by adaptation ability.

One might further argue that the observed relationship between motor performance and adaptation indicators is artefactual: it would also emerge if this relationship did not exist on a subject-to-subject basis, as long as the patients as a group would exhibit both a poorer motor performance and a poorer adaptation than controls as a group. Figure 4 illustrates for one performance and one adaptation score that this was not the case: patients and controls overlapped with respect to AI, as well as with respect to MT. More importantly, the multiple regression between motor performance and adaptation indicators remained significant even when the analysis was limited to the patient group only $\left(R_{\mathrm{AI}}^{2}=0.6494^{*}, R_{\mathrm{AR}}^{2}=0.6515^{*}, R_{\mathrm{AT}}^{2}=0.2955\right.$, and $\left.R_{\mathrm{DA}}^{2}=0.7487^{* *}\right)$.

Since the above analyses included five different measures of motor performance, we explored which of them are crucial for the significance pattern in Table 3, by replicating the regression analyses with different subsets of those measures. We found that the significance pattern

Table 3 Outcome of linear regression analyses

\begin{tabular}{llll}
\hline$a$ & $\operatorname{Var}_{G}$ & $\operatorname{Var}_{J}$ & $\operatorname{Var}_{P}$ \\
\hline AI & 0.0123 & $0.3530^{* * *}$ & $0.2600 * *$ \\
AR & 0.0001 & $0.1687^{*}$ & $0.3883 * * *$ \\
AT & 0.0449 & 0.0744 & $0.1719 *$ \\
DA & 0.0327 & $0.3168 * * *$ & $0.1691 *$ \\
\hline
\end{tabular}

The total variance of each adaptation indicator was partitioned into a component $\operatorname{Var}_{G}$ shared with subject group but not with motor performance, a component $\operatorname{Var}_{J}$ shared jointly with group and motor performance, and a component $\operatorname{Var}_{P}$ shared with motor performance alone. Symbols have the same meaning as in Table 2 persisted as long as the analyses included MT and CA, or MT and RT. We therefore concluded that MT, CA, and RT are indicators of adaptive success. These are the same three measures which differed significantly between patients and controls, and which correlated significantly with patients' ataxia scores (see Table 2). As an example, Fig. 4 depicts the relationship between MT and AI in both subject groups.

\section{Discussion}

The present study evaluated adaptive success and motor performance in patients with cerebellar cortical degeneration, and in healthy control subjects. In accordance with literature (Deuschl et al. 1996; Diedrichsen et al. 2005; Gauthier et al. 1979; Martin et al. 1996; Maschke et al. 2004; Tseng et al. 2007; Weiner et al. 1983), we found that patients adapted less well than controls. Also in accordance with previous work (Martin et al. 1996; Maschke et al. 2004; Weiner et al. 1983), the deficit was not limited to the adaptation phase, but rather continued undiminished

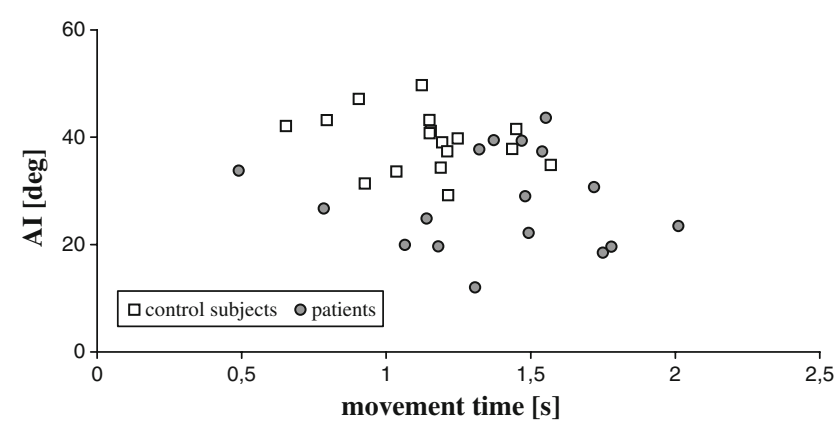

Fig. 4 Relationship between movement time and adaptive improvement AI in patients (gray) and controls (white). Each symbol represents one subject 
throughout the retention and transfer into the de-adaptation phase. Such a persistence of the deficit is interesting, as it allows an insight into the underlying pathology. It is thought that adaptive improvement is based on two distinct processes, a recalibration of sensory-to-motor transformation rules, and strategic control by anticipations, associative stimulus-response pairings, and other workaround schemes; in contrast, retention, transfer, and de-adaptation are thought to reflect recalibration alone (Bock 2005; McNay and Willingham 1998; Redding 1996). If so, the persistence of an adaptation deficit in our patients would indicate that recalibration but not strategic control is impaired by cerebellar degeneration.

Besides adaptive success, movement performance was also degraded in our patients. In accordance with literature, we found an increase of reaction and movement time, less efficient online error corrections, but normal peak movement velocity (Bonnefoi-Kyriacou et al. 1998; Holmes 1917; Hore et al. 1991; Tseng et al. 2007). Previous work further reported an increase of movement variability (Martin et al. 1996; Timmann et al. 1999; Tseng et al. 2007) which didn't reach statistical significance in our study. This is probably so because our patients' ataxia was relatively mild (see Table 1); cerebellar areas related to upper-limb ataxia (Martin et al. 1996) may not have been profoundly affected in all our patients.

The main purpose of the present study was to scrutinize the interrelation between patients' deficits of adaptation and of motor performance. We therefore partitioned the variance of each adaptation indicator into three components. $\operatorname{Var}_{G}$ was related to subject group but not motor performance, and did not reach statistical significance for any indicator; we therefore have no evidence for the existence of cerebellar circuitry dedicated to adaptation but not to motor performance. $\operatorname{Var}_{J}$ was related jointly to group and motor performance, and $\operatorname{Var}_{P}$ to motor performance alone. The latter two components were significant for most or all adaptation indicators, which suggests that cerebellar and extracerebellar brain regions involved in the monitoring and control of movements also contributed towards adaptive success. This contribution could be interpreted in two ways: the respective brain regions could be involved in motor performance alone and send their output to adaptive mechanisms located elsewhere, or they could be involved in both functions, motor performance and adaptation.

The above conclusions are pertinent to the two positions on the role of the cerebellum, as outlined in the Introduction. Our findings are in accordance with one of the views, which holds that the cerebellum provides performance-related signals as a prerequisite for adaptation. They also agree with a compromise between both views, which posits that adaptation and motor performance are processed in identical or highly interlinked cerebellar structures. However, our findings do not support the pure version of the other view, according to which the cerebellum contains circuitry dedicated to adaptation but not motor performance.

Our analyses indicate that three measures of motor performance were associated with adaptive success. One of them represented the efficiency of online error corrections. The simple correlation between adaptation indicators and this measure ranged between 0.46 and 0.71 , which is similar to the correlations reported before (Tseng et al. 2007). The correlations were significant in the present work $(p<0.01)$ but not in the previous study, possibly because the variables were defined somewhat differently, and/or because the sample size was larger in the present $(n=2 \times 17)$ than in the previous study $(n=2 \times 7)$. The existence of sizable correlations should not be taken as evidence that adaptation is driven by online response corrections; this surmise has been convincingly refuted before, and replaced by the view that adaptation is driven by sensory prediction errors (Tseng et al. 2007). Instead, the observed correlation could reflect the dependence both of adaptive success and of online corrections on a common causal factor, e.g., cerebellar function.

The present study not only assessed the role of the cerebellum in sensorimotor adaptation, but also provided evidence for adaptive mechanisms located outside the cerebellum. As stated above, the significance of $\operatorname{Var}_{P}$ suggests that extracerebellar brain regions related to motor performance either were involved in adaptation as well, or sent their output to other extracerebellar areas dedicated to adaptation. Reasonable candidates for such areas are the inferior parietal and the dorsal premotor cortex: a recent neuroimaging study which controlled for error-related brain activity found significant extracerebellar activation only in these two areas (Girgenrath et al. 2007).

Acknowledgments This work was supported by DFG grant Bo 649/ 8, and by grant 50WB9942 from the German Ministry for Education and Research, both awarded to O. B. Responsibility for contents rests with the authors.

Open Access This article is distributed under the terms of the Creative Commons Attribution Noncommercial License which permits any noncommercial use, distribution, and reproduction in any medium, provided the original author(s) and source are credited.

\section{References}

Albus J (1971) A theory of cerebellar function. Math Biosci 10:25-61 Bloedel J, Bracha V (1997) Duality of cerebellar motor and cognitive functions. Int Rev Neurobiol 41:613-634

Bock O (2005) Components of sensorimotor adaptation in young and elderly subjects. Exp Brain Res 160:259-263

Bock O, Girgenrath M (2006) Relationship between sensorimotor adaptation and cognitive functions in younger and older subjects. Exp Brain Res 169:400-406 
Bock O, Schneider S, Bloomberg J (2001) Conditions for interference versus facilitation during sequential sensorimotor adaptation. Exp Brain Res 138:359-365

Bonnefoi-Kyriacou B et al (1998) Spatio-temporal and kinematic analysis of pointing movements performed by cerebellar patients with limb ataxia. Exp Brain Res 119:460-466

Deuschl G et al (1996) Adaption motor learning of arm movements in patients with cerebellar disease. J Neurol Neurosurg Psychiatry 60:515-519

Diedrichsen $\mathbf{J}$ et al (2005) Cerebellar involvement in anticipating the consequences of self-produced actions during bimanual movements. J Neurophysiol 93:801-812

Dimitrova A et al (2006) Probabilistic 3D MRI atlas of the human cerebellar dentate/interposed nuclei. Neuroimage 30:12-25

Flament D et al (1996) Functional magnetic resonance imaging of cerebellar activation during the learning of a visuomotor dissociation task. Hum Brain Map 4:210-226

Gauthier GM et al (1979) Visual-motor adaptation: quantitative demonstration in patients with posterior fossa involvement. Arch Neurol 36:155-160

Gerwig M et al (2003) Comparison of eyeblink conditioning in patients with superior and posterior inferior cerebellar lesions. Brain 126:71-94

Girgenrath M et al (2007) An fMRI study of brain activation in a visual adaptation task: activation limited to sensory guidance. Exp Brain Res 184:561-569

Graydon F et al (2005) Learning-related fMRI activation associated with a rotational visuo-motor transformation. Cognit Brain Res 22:373-383

Holmes G (1917) The symptoms of acute cerebellar injuries due to gunshot injuries. Brain 40:461-535

Hore $\mathbf{J}$ et al (1991) Cerebellar dysmetria at the elbow, wrist, and fingers. J Neurophysiol 65:563-571

Imamizu $\mathrm{H}$ et al (2000) Human cerebellar activity reflecting an acquired internal model of a new tool. Nature 403:192-195

Krakauer J, Ghilardi M-F, Mentis M, Barnes A, Veytsman M, Eidelberg D, Ghez C (2004) Differential cortical and subcortical activations in learning rotations and gains for reaching: a PET study. J Neurophysiol 91:924-933
Krebs H et al (1998) Robot-aided functional imaging: application to a motor learning study. Hum Brain Map 6:59-72

Lang W et al (1988) DC-potential shifts and regional cerebral blood flow reveal frontal cortex involvement in human visuomotor learning. Exp Brain Res 71:353-364

Marr D (1969) A theory of cerebellar cortex. J Physiol 202:437-470

Martin TA et al (1996) Throwing while looking through prisms. I. Focal olivocerebellar lesions impair adaptation. Brain 119:11831198

Maschke M et al (2000) Involvement of the human medial cerebellum in long-term habituation of the acoustic startle response. Exp Brain Res 133:359-367

Maschke M et al (2004) Hereditary cerebellar ataxia progressively impairs force adaptation during goal-directed arm movements. J Neurophysiol 91:230-238

McNay EC, Willingham DB (1998) Deficit in learning of a motor skill requiring strategy, but not of perceptualmotor recalibration, with aging. Learn Mem 4:411-420

Redding G (1996) Adaptive spatial alignment and strategic perceptual-motor control. J Exp Psychol Hum Percept Perform 22:379394

Schmitz-Hubsch T et al (2006) Scale for the assessment and rating of ataxia: development of a new clinical scale. Neurology 66:17171720

Timmann D et al (2000) Clasically conditioned withdrawal reflex in cerebellar patients. 1. Impaired conditioned responses. Exp Brain Res 130:453-470

Timmann D et al (1999) Failure of cerebellar patients to time finger opening precisely causes ball high-low inaccuracy in overarm throws. J Neurophysiol 82:103-114

Tseng Y et al (2007) Sensory prediction errors drive cerebellum-dependent adaptation of reaching. J Neurophysiol 98(1):54-62

Weiner MJ et al (1983) Adaptation to lateral displacement of vision in patients with lesions of the central nervous system. Neurology 33:766-772

Woodruff-Pak D (1997) Classical conditioning. Int Rev Neurobiol 41:341-366 